Document downloaded from:

http://hdl.handle.net/10251/176156

This paper must be cited as:

Cantó Colomina, R.; Moll López, SE.; Ricarte Benedito, B.; Urbano Salvador, AM. (2020). A direct method to obtain a realization of a polynomial matrix and its applications. Revista de la Real Academia de Ciencias Exactas Físicas y Naturales Serie A Matemáticas. 114(2):1-15. https://doi.org/10.1007/s13398-020-00819-1

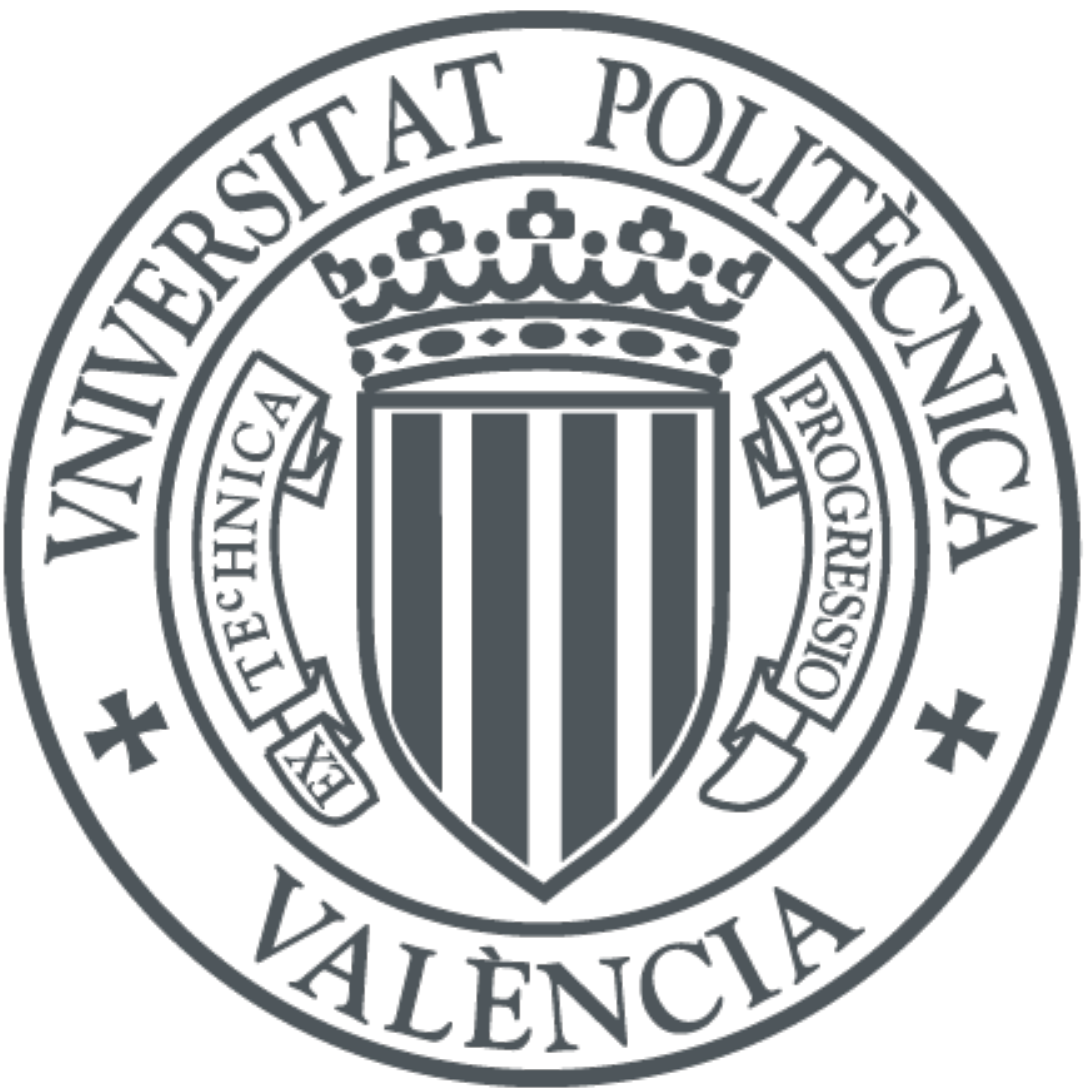

The final publication is available at

https://doi.org/10.1007/s13398-020-00819-1

Copyright Springer-Verlag

Additional Information 


\title{
A direct method to obtain a realization of a polynomial matrix and its applications
}

\author{
Rafael Cantó $^{1}$ - Santiago Moll ${ }^{2}$ - Beatriz \\ Ricarte $^{1}$ • Ana María Urbano ${ }^{1}$.
}

Received: date / Accepted: date

\begin{abstract}
In this paper we present a Silverman-Ho algorithm-based method to obtain a realization of a polynomial matrix. This method provides the final formulation of a minimal realization directly from a full rank factorization of a specific given matrix. Also, some classical problems in control theory such as model reduction in singular systems or the positive realization problem in standard systems are solved with this method.
\end{abstract}

Keywords Full rank factorization · Polynomial matrices · Applied Mathematics - Minimal realization

Mathematics Subject Classification (2010) 15A23 - 93B15 93 B20

\section{Introduction}

A matrix with polynomial entries, or equivalently a polynomial with matrix coefficients is called a polynomial matrix. Polynomial matrices can be found

An earlier version of this paper was presented at the Conference "Linear Algebra, Matrix Analysis and Applications. ALAMA2018", held in Sant Joan d'Alacant on May/June 2018.

Work supported by the Spanish DGI grant MTM2017-85669-P-AR.

Rafael Cantó

rcanto@mat.upv.es

Santiago Moll

sanmoll@mat.upv.es

Beatriz Ricarte

bearibe@mat.upv.es

Ana María Urbano

amurbano@mat.upv.es

${ }^{1}$ Institut de Matemàtica Multidisciplinar, Universitat Politècnica de València, Spain.

${ }^{2}$ Institut de Matemàtica Pura i Aplicada, Universitat Politècnica de València, Spain. 
in a large variety of applications in science and engineering. For instance, second degree polynomial matrices arise in the control of large flexible space structures, earthquake engineering problems, control of mechanical multi-body systems, stabilization of damped gyroscopic systems, robotics and vibration control in structural dynamics. Third degree polynomial matrices are often used in aero-acoustics, and quartic polynomial matrices appear in fluid mechanics when studying the spatial stability of the Orr-Sommerfeld equation $[9]$.

Given a polynomial matrix $P(s) \in \mathbb{R}^{m \times p}$,

$$
P(s)=W_{0}+W_{1} s+\cdots+W_{t-1} s^{t-1}
$$

where $W_{i} \in \mathbb{R}^{m \times p}, i=0,1, \ldots, t-1$, there exist matrices $N, B$ and $C$, with $N$ nilpotent, such that $P(s)=C(s N-I)^{-1} B$ [7, Lemma 2-6.2]. Matrices $(N, I, B, C)$ are known as a realization of $P(s)$. The Silverman-Ho algorithm was originally employed to compute a minimal realization of rational transfer matrices (further details can be found in references [10] and [16] or in [1]), where minimal means that the realization has the minimum dimension. Later, the Silverman-Ho algorithm was adapted to compute a minimal realization $(N, I, B, C)$ of polynomial transfer matrices [7, pp. 63]. This algorithm is applied to an unspecified full rank factorization $F G$ of a block matrix $M_{0} \in \mathbb{R}^{t m \times t p}$ consisting of matrices $W_{i}$ (see Section 2). Matrices $F$ and $G$ are used to computed the nilpotent matrix $N$ by means of products of these matrices, their transpose matrices and inverse matrices of some of these products, which, from a numerical point of view, could make this method quite unstable and lead to a non-nilpotent matrix.

In this paper a direct method to derive a realization of $P(s)$ that computes the nilpotent matrix $N$ directly from a specific full rank factorization is developed, avoiding the computation of inverse matrices and products, this being the main disadvantage of the Silverman-Ho algorithm. The method presented in this paper is applied to an initial block upper triangular matrix $W=M_{0} P$, where $P$ is a block permutation matrix reversing the order of the column blocks of $M_{0}$, and obtains a full rank factorization $\bar{G} \bar{U}$, considering its reduced echelon form (that can be obtained by using different methods such as the Gaussian elimination method with partial or complete pivoting [8]).

Our full rank factorization $W=\bar{G} \bar{U}$ satisfies that $\bar{U}$ is an upper reduced echelon form given by

$$
\bar{U}=\left[\begin{array}{cc|cc|c|cc}
I_{r_{1}} & \bar{U}^{(1,1)} & O & \bar{U}^{(2,1)} & \cdots & O & \bar{U}^{(t, 1)} \\
O & O & I_{r_{2}} & \bar{U}^{(2,2)} & \cdots & O & \bar{U}^{(t, 2)} \\
\vdots & \vdots & \vdots & \vdots & & \vdots & \vdots \\
O & O & O & O & \cdots & I_{r_{t}} & \bar{U}^{(t, t)}
\end{array}\right]
$$

Finally, selecting in a specific way certain columns of $\bar{U}$, matrices $B$ and $N$ can be constructed, while matrix $C$ is the submatrix formed by the first $m$ rows of $G$. Therefore, the main advantage of the proposed method over the 
Silverman-Ho algorithm is its simplicity for obtaining a realization of $P(s)$ with a lower computational cost.

Also, it is worth noting that polynomial matrices also appear in the inputoutput representation of singular systems, since any rational matrix may be represented by the sum of a strictly proper rational matrix and a polynomial matrix [7].

The realization problem of strictly proper rational matrices has been widely studied and, specifically in the positive case, it has been proved that it is not always possible to find a positive realization with the known algorithms $[5,11$, 13]. Hence, an alternative method is here presented giving the conditions to compute a positive realization.

Throughout this work, given a matrix $A \in \mathbb{R}^{n \times m}, A\left(i_{1}: i_{2}, j_{1}: j_{2}\right)$ will denote the submatrix of $A$ with rows $\left\{i_{1}, i_{1}+1, i_{1}+2, \ldots, i_{2}\right\}$ and columns $\left\{j_{1}, j_{1}+1, j_{1}+2, \ldots, j_{2}\right\}$. If the submatrix has all rows (resp. columns) of $A$, then it is denoted by $A\left(:, j_{1}: j_{2}\right)$ (resp. $A\left(i_{1}: i_{2},:\right)$ ).

\section{Preliminaries and previous results}

First in this section, the Silverman-Ho algorithm is introduced.

\section{Algorithm 1.}

Consider the polynomial matrix $P(s) \in \mathbb{R}^{m \times p}$ of order $t-1$ given by equation (1).

Step 1. Define the matrices

$$
\begin{gathered}
M_{0}=\left[\begin{array}{ccccc}
-W_{0} & -W_{1} & \cdots & -W_{t-2} & -W_{t-1} \\
-W_{1} & -W_{2} & \cdots & -W_{t-1} & O \\
\vdots & \vdots & & \vdots & \vdots \\
-W_{t-2} & -W_{t-1} & \cdots & O & O \\
-W_{t-1} & O & \cdots & O & O
\end{array}\right] \in \mathbb{R}^{t m \times t p} \\
M_{1}=\left[\begin{array}{ccccc}
-W_{1} & -W_{2} & \cdots & -W_{t-1} & O \\
-W_{2} & -W_{3} & \cdots & O & O \\
\vdots & \vdots & & \vdots & \vdots \\
-W_{t-1} & O & \cdots & O & O \\
O & O & \cdots & O & O
\end{array}\right] \in \mathbb{R}^{t m \times t p}
\end{gathered}
$$

Step 2. Consider a full rank decomposition $M_{0}=F G$, where $F \in \mathbb{R}^{t m \times n}$ and $G \in \mathbb{R}^{n \times t p}$ have full column and full row rank, respectively.

Step 3. Matrix $B$ and $C$ are equal to the first $p$ columns of $G$ and the first $m$ rows of $F$, respectively. Finally, $N=\left(F^{T} F\right)^{-1} F^{T} M_{1} G^{T}\left(G G^{T}\right)^{-1}$.

Matrices $(N, I, B, C)$ determine a realization of $P(s)$. 
Now, a procedure to obtain a full rank factorization $\bar{G} \bar{U}$ of certain singular block upper triangular matrix is presented, in such a way that $\bar{U}$ has the structure given by (2).

\section{Procedure 1.}

Consider the following block upper triangular matrix

$$
M=\left[\begin{array}{ccccc}
M_{11} & M_{12} & \cdots & M_{1 t-1} & M_{1 t} \\
O & M_{11} & \cdots & M_{1 t-2} & M_{1 t-1} \\
\vdots & \vdots & & \vdots & \vdots \\
O & O & \cdots & M_{11} & M_{12} \\
O & O & \cdots & O & M_{11}
\end{array}\right] \in \mathbb{R}^{t m \times t p}
$$

with $\operatorname{rank}(M)=q$.

Step 1. Obtain $R$, the reduced echelon form of $M$.

Step 2. Obtain the full rank factorization $M=G U$ such that $G$ is the matrix formed by the linearly independent columns of $M$ and $G$ is the matrix formed by the nonzero rows of $R$. If $U$ has the shape of (2), then $\bar{G}=G, \bar{U}=U$ and $P=I$. Otherwise, go to the following step.

Step 3. Find a permutation matrix $P=\operatorname{diag}\left(P_{1} P_{1} \ldots P_{1}\right) \in \mathbb{R}^{t p \times t p}$ such that the matrix $U P$ has the leading 1's in the first columns of each column blocks. If $U P$ has the shape of (2), then $M P=\bar{G} \bar{U}$, where $\bar{G}=G$ and $\bar{U}=U P$. In other case, go to the following step.

Step 4. Find a permutation matrix $Z$ such that $\bar{U}=Z U P$ follows the structure (2). Then, $M P=\bar{G} \bar{U}$, where $\bar{G}=G Z^{T}$ and $\bar{U}=Z U P$.

Concluding, this procedure computes the permutation matrices $P$ and $Z$, and two matrices $\bar{G}$ and $\bar{U}$, such that $M P=\bar{G} \bar{U}$, where $\bar{G} \in \mathbb{R}^{t m \times q}, \bar{U} \in$ $\mathbb{R}^{q \times t p}$ is an upper reduced echelon matrix with the same form than (2) and $\operatorname{rank}(\bar{G})=\operatorname{rank}(\bar{U})=q$.

Example 1 Consider the block matrix

$$
M=\left[\begin{array}{lll}
M_{1} & M_{2} & M_{3} \\
O & M_{1} & M_{2} \\
O & O & M_{1}
\end{array}\right]=\left[\begin{array}{lll|lll|lll}
0 & 1 & 2 & 1 & 2 & 6 & 1 & 2 & 3 \\
0 & 2 & 4 & 0 & 1 & 4 & 2 & 1 & 0 \\
0 & 1 & 2 & 1 & 0 & 2 & 1 & 0 & 2 \\
\hline 0 & 0 & 0 & 0 & 1 & 2 & 1 & 2 & 6 \\
0 & 0 & 0 & 0 & 2 & 4 & 0 & 1 & 4 \\
0 & 0 & 0 & 0 & 1 & 2 & 1 & 0 & 2 \\
\hline 0 & 0 & 0 & 0 & 0 & 0 & 0 & 1 & 2 \\
0 & 0 & 0 & 0 & 0 & 0 & 0 & 2 & 4 \\
0 & 0 & 0 & 0 & 0 & 0 & 0 & 1 & 2
\end{array}\right] .
$$


Its reduced echelon form is

$$
R=\left[\begin{array}{lll|lll|lll}
0 & 1 & 2 & 0 & 0 & 1 & 0 & 0 & 0 \\
0 & 0 & 0 & 1 & 0 & 1 & 0 & 0 & 0 \\
0 & 0 & 0 & 0 & 1 & 2 & 0 & 0 & 0 \\
0 & 0 & 0 & 0 & 0 & 0 & 1 & 0 & 0 \\
0 & 0 & 0 & 0 & 0 & 0 & 0 & 1 & 0 \\
0 & 0 & 0 & 0 & 0 & 0 & 0 & 0 & 1 \\
0 & 0 & 0 & 0 & 0 & 0 & 0 & 0 & 0 \\
0 & 0 & 0 & 0 & 0 & 0 & 0 & 0 & 0 \\
0 & 0 & 0 & 0 & 0 & 0 & 0 & 0 & 0
\end{array}\right]
$$

Therefore, the full rank factorization $M=G U$ is given by

$$
M=\left[\begin{array}{llllll}
1 & 1 & 2 & 1 & 2 & 3 \\
2 & 0 & 1 & 2 & 1 & 0 \\
1 & 1 & 0 & 1 & 0 & 2 \\
0 & 0 & 1 & 1 & 2 & 6 \\
0 & 0 & 2 & 0 & 1 & 4 \\
0 & 0 & 1 & 1 & 0 & 2 \\
0 & 0 & 0 & 0 & 1 & 2 \\
0 & 0 & 0 & 0 & 2 & 4 \\
0 & 0 & 0 & 0 & 1 & 2
\end{array}\right]\left[\begin{array}{lll|lll|lll}
0 & 1 & 2 & 0 & 0 & 1 & 0 & 0 & 0 \\
0 & 0 & 0 & 1 & 0 & 1 & 0 & 0 & 0 \\
0 & 0 & 0 & 0 & 1 & 2 & 0 & 0 & 0 \\
0 & 0 & 0 & 0 & 0 & 0 & 1 & 0 & 0 \\
0 & 0 & 0 & 0 & 0 & 0 & 0 & 1 & 0 \\
0 & 0 & 0 & 0 & 0 & 0 & 0 & 0 & 1
\end{array}\right]=G U .
$$

Since $U$ has not the shape of (2), we need to permute some of its columns. Consider the permutation matrix $P=\operatorname{diag}\left(P_{1} P_{1} P_{1}\right)$ where $P_{1}=\left[\begin{array}{lll}0 & 1 & 0 \\ 1 & 0 & 0 \\ 0 & 0 & 1\end{array}\right]$. Then,

$$
\tilde{U}=U P=\left[\begin{array}{lll|lll|lll}
1 & 0 & 2 & 0 & 0 & 1 & 0 & 0 & 0 \\
0 & 0 & 0 & 0 & 1 & 1 & 0 & 0 & 0 \\
0 & 0 & 0 & 1 & 0 & 2 & 0 & 0 & 0 \\
0 & 0 & 0 & 0 & 0 & 0 & 0 & 1 & 0 \\
0 & 0 & 0 & 0 & 0 & 0 & 1 & 0 & 0 \\
0 & 0 & 0 & 0 & 0 & 0 & 0 & 0 & 1
\end{array}\right]
$$

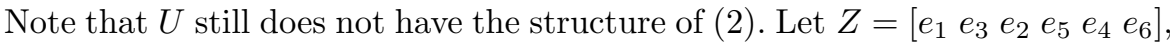
being $e_{i}, i=1,2, \ldots, 6$, the canonical vectors. With the matrices

$$
\bar{G}=G Z^{T}=\left[\begin{array}{llllll}
1 & 2 & 1 & 2 & 1 & 3 \\
2 & 1 & 0 & 1 & 2 & 0 \\
1 & 0 & 1 & 0 & 1 & 2 \\
0 & 1 & 0 & 2 & 1 & 6 \\
0 & 2 & 0 & 1 & 0 & 4 \\
0 & 1 & 0 & 0 & 1 & 2 \\
0 & 0 & 0 & 1 & 0 & 2 \\
0 & 0 & 0 & 2 & 0 & 4 \\
0 & 0 & 0 & 1 & 0 & 2
\end{array}\right], \quad \bar{U}=Z U P=Z \tilde{U}=\left[\begin{array}{lll|lll|lll}
1 & 0 & 2 & 0 & 0 & 1 & 0 & 0 & 0 \\
0 & 0 & 0 & 1 & 0 & 2 & 0 & 0 & 0 \\
0 & 0 & 0 & 0 & 1 & 1 & 0 & 0 & 0 \\
0 & 0 & 0 & 0 & 0 & 0 & 1 & 0 & 0 \\
0 & 0 & 0 & 0 & 0 & 0 & 0 & 1 & 0 \\
0 & 0 & 0 & 0 & 0 & 0 & 0 & 0 & 1
\end{array}\right],
$$


we have the full rank factorization

$$
M P=\left(G Z^{T}\right)(Z U P)=\bar{G} \bar{U}
$$

where $\bar{U}$ follows the structure (2).

\section{Factorization of polynomial matrices}

Given a polynomial matrix $P(s) \in \mathbb{R}^{m \times p}$, in this section we present a constructive procedure to determine a realization of $P(s)$, namely, matrices $N \in \mathbb{R}^{n \times n}$, $B \in \mathbb{R}^{n \times p}$ and $C \in \mathbb{R}^{m \times n}$, such that

$$
P(s)=C(s N-I)^{-1} B=-C B-C N B s-\ldots-C N^{t-1} B s^{t-1}
$$

where $N$ is a nilpotent matrix with nilpotent index equal to $t$. As mentioned in the introduction, the method presented here can be considered as an interesting improvement of the Silverman-Ho algorithm [7, pp. 63]. We can suppose, without loss of generality, that $m \geq p$. In other case, we can work with the polynomial transfer matrix $P^{T}(s)$, in such a way that if $(N, I, B, C)$ is a realization of $P(s)$, then $\left(N^{T}, I, C^{T}, B^{T}\right)$ is a realization of $P^{T}(s)$.

\section{Procedure 2.}

Let $P(s)=W_{0}+W_{1} s+\ldots+W_{t-1} s^{t-1} \in \mathbb{R}^{m \times p}$ with $W_{i} \in \mathbb{R}^{m \times p}, i=$ $0,1, \ldots, t-1$ be a polynomial matrix.

Step 1. Compute the block matrix $W$ given by

$$
W=\left[\begin{array}{ccccc}
-W_{t-1} & -W_{t-2} & \cdots & -W_{1} & -W_{0} \\
O & -W_{t-1} & \cdots & -W_{2} & -W_{1} \\
\vdots & \vdots & & \vdots & \vdots \\
O & O & \cdots & -W_{t-1} & -W_{t-2} \\
O & O & \cdots & O & -W_{t-1}
\end{array}\right] \in \mathbb{R}^{t m \times t p}
$$

Step 2. Apply Procedure 1 to obtain the full rank factorization

$$
W P=\bar{G} \bar{U}=\left[\begin{array}{c}
\bar{G}_{1} \\
\bar{G}_{2} \\
\vdots \\
\bar{G}_{t-1} \\
\bar{G}_{t}
\end{array}\right]\left[\bar{U}_{1} \bar{U}_{2} \cdots \bar{U}_{t-1} \bar{U}_{t}\right]
$$

where $\bar{G}_{i} \in \mathbb{R}^{m \times n}$ and $\bar{U}_{i} \in \mathbb{R}^{n \times p}$ for $i=1,2, \ldots, t$, with $n=\operatorname{rank}(W)$ and $\bar{U}$ is an upper reduced echelon form with the same structure than (2). 
Step 3. Define

$$
\begin{aligned}
B & =\bar{U}(:,(t-1) p+1: t p), \quad C=\bar{G}(1: m,:) \\
q_{t-1} & =\operatorname{rank}(\bar{U}(:, 1: p)) \\
q_{t-i} & =\operatorname{rank}(\bar{U}(:, 1: i p))-\operatorname{rank}(\bar{U}(:, 1:(i-1) p)), \text { for } i=2,3, \ldots, t . \\
N & =\left[O_{n \times q_{t-1}} \bar{U}\left(:, 1: q_{t-2}\right) \bar{U}\left(:, p+1: p+q_{t-3}\right) \ldots\right. \\
& \left.\ldots \bar{U}\left(:,(t-2) p+1:(t-2) p+q_{0}\right)\right]
\end{aligned}
$$

Step 4. - If $P=I$ then, $(N, I, B, C)$ constitutes a minimal realization of $P(s)$.

- If $P=\operatorname{diag}\left(P_{1} P_{1} \ldots P_{1}\right)$ then, $\left(N, I, B P_{1}^{T}, C\right)$ constitutes a minimal realization of $P(s)$.

Remark 1 It bears noting that:

1. The subindexes $q_{t-i}, i=1,2, \ldots, t$, are directly obtained from the number of leading 1 's of the corresponding blocks of $\bar{U}$. Hence, if $\bar{U} \in \mathbb{R}^{n \times p t}$ is the matrix

$$
\bar{U}=\left[\begin{array}{cc|cc|c|cc|cc}
I_{q_{t-1}} & \bar{U}^{(1,1)} & O & \bar{U}^{(2,1)} & \cdots & O & \bar{U}^{(t-1,1)} & O & \bar{U}^{(t, 1)} \\
O & O & I_{q_{t-2}} & \bar{U}^{(2,2)} & \ldots & O & \bar{U}^{(t-1,2)} & O & \bar{U}^{(t, 2)} \\
O & O & O & O & \ldots & O & \bar{U}^{(t-1,3)} & O & \bar{U}^{(t, 3)} \\
\vdots & \vdots & \vdots & \vdots & & \vdots & \vdots & \vdots & \vdots \\
O & O & O & O & \ldots & I_{q_{1}} & \bar{U}^{(t-1, t-1)} & O & \bar{U}^{(t, t-1)} \\
O & O & O & O & \cdots & O & O & I_{q_{0}} & \bar{U}^{(t, t)}
\end{array}\right] .
$$

$\bar{G}$ is given from the $n$ linearly independent columns of $W$, which are $q_{t-1}$ from the first column block, $q_{t-2}$ from the second column block, and so on until the last $q_{0}$ linearly independent columns from the last column block of $W$. Note that $q_{t-1}+q_{t-2}+\cdots+q_{0}=n$. Consequently, we only need to save the column indexes of $\bar{U}$ with leading 1 's.

2. By construction $N$ is nilpotent, with nilpotent index equal to $t$.

Proposition 1 The matrices $N, I, B$ and $C$ given by Procedure 2 constitute a minimal realization of the polynomial transfer matrix $P(s)=W_{0}+W_{1} s+$ $\ldots+W_{t-1} s^{t-1}$, where $W_{i} \in \mathbb{R}^{m \times p}, i=0,1, \ldots, t-1$.

Proof First suppose that $P=I$. Then, from the structure of matrices $B, C$ and $N$, these equations are satisfied:

$$
C B=-W_{0}, \quad C N^{i} B=-W_{i} \quad i=1,2, \ldots, t-1 .
$$

Hence $(N, I, B, C)$ is a realization of $P(s)$ and it will be minimal if the corresponding system is controllable and observable [7]. A system is controllable 
if the rank of its controllability matrix is equal to the dimension $n$ of the realization. For $i=1,2, \ldots, t-1$ we have

$$
N^{i} B=\left[\begin{array}{cc|c}
O & \bar{U}^{(t-i, 1)}\left(:, 1: q_{i-1}-q_{i}\right) & \star \\
O & \bar{U}^{(t-i, 2)}\left(:, 1: q_{i-1}-q_{i}\right) & \star \\
\vdots & \vdots & \vdots \\
I_{q_{i}} & \bar{U}^{(t-i, t-i)}\left(:, 1: q_{i-1}-q_{i}\right) & \star \\
O & O & \vdots \\
\vdots & \vdots & O \\
O & O &
\end{array}\right],
$$

where the partition by rows is given by $q_{t-1}, q_{t-2}, \ldots, q_{i}, q_{i-1}, \ldots, q_{0}$. Then

$$
\begin{aligned}
\operatorname{rank}\left(\left[B N B N^{2} B \ldots N^{t-1} B\right]\right) & =q_{0}+q_{1}+q_{2}+\cdots+q_{t-2}+q_{t-1} \\
& =\operatorname{rank}(\bar{U})=n .
\end{aligned}
$$

Therefore, the system given by $(N, I, B, C)$ is controllable.

On the other hand, a system is observable if the rank of its observability matrix $\mathcal{O}$ is also equal to $n$. Since

$$
\mathcal{O}=\left[\begin{array}{c}
C \\
C N \\
C N^{2} \\
\vdots \\
C N^{t-2} \\
C N^{t-1}
\end{array}\right]
$$

and

$$
C N^{i}=\left[\begin{array}{c}
O_{n \times q_{t-1}} \\
\vdots \\
\left.O_{n \times q_{t-i}}\right) \\
\bar{G}_{1}\left(:, 1 q_{t-1}\right) \\
\star \\
\bar{G}_{1}\left(:, q_{t-1}+1: q_{t-1}+q_{t-2}\right) \\
\vdots \\
\bar{G}_{1}\left(:, \sum_{j=1}^{t-i+1} q_{t-i}+1: \sum_{j=1}^{t-i} q_{t-j}\right) \\
\star
\end{array}\right]^{T}
$$

$\operatorname{rank}(\mathcal{O})=n$, and the system given by $(N, I, B, C)$ is observable. Consequently, this realization is minimal.

Now, suppose that $P=\operatorname{diag}\left(P_{1} P_{1} \ldots P_{1}\right)$ and consider the transfer matrix

$$
\begin{aligned}
\bar{P}(s)=P(s) P_{1} & =\left(W_{0} P_{1}\right)+\left(W_{1} P_{1}\right) s+\cdots+\left(W_{t-1} P_{1}\right) s^{t-1} \\
& =\bar{W}_{0}+\bar{W}_{1} s+\cdots+\bar{W}_{t-1} s^{t-1}
\end{aligned}
$$


and the block matrix $\bar{W}=W P$. Applying Procedure 2 we have the full rank factorization

$$
\bar{W} \bar{P}=\bar{G} \bar{U}
$$

where $\bar{P}=I$ and $\bar{U}$ has the structure given by (2). From this factorization we obtain matrices $\left(N, I_{n}, B, C\right)$, which compose a minimal realization of the polynomial matrix $\bar{P}(s)$, as just demonstrated. As, for $i=0,1, \ldots, t-1$, it is verifies

$$
C N^{i} B=-\bar{W}_{i}=-W_{i} P_{1} \Longrightarrow C N^{i} B P_{1}^{T}=-W_{i}
$$

then $\left(N, I_{n}, B P_{1}^{T}, C\right)$ is a minimal realization of $P(s)$.

Remark 2 It is worth noting that if the complete quasi-Gauss elimination process is used to obtain the initial full rank factorization, it is possible for illconditioned matrices to have a range greater than it should have. In this case a minimal realization is not obtained, since $\bar{U}$ will have more leading $1^{\prime} s$ than it really should have, overestimating some of the parameters $q_{i}$. As $B$ and $N$ are directly obtained from $\bar{U}$, the corresponded realization will be controllable by construction but, an overestimated parameter $q_{i}$ implies that some of the linear dependent columns of $W$ are being selected for computing $\bar{G}$ and hence $C$, implying that this realization will not be observable.

Let us look at an example where the matrices of coefficients of the polynomial transfer matrix $P(s)$ are obtained from Hilbert matrices. Remind that, in linear algebra, a Hilbert matrix is a square matrix with entries being the unit fractions

$$
H_{i j}=\frac{1}{i+j-1} .
$$

The Hilbert matrices are canonical examples of ill-conditioned matrices, being notoriously difficult to use in numerical computation. Therefore, the following purely academic example has the purpose to just highlight the previous remark.

Example 2 Consider the polynomial matrix $P(s)=W_{0}+W_{1} s+W_{2} s^{2}$, with

$$
\begin{aligned}
& W_{0}=-\left(\operatorname{hilb}(15)-0.1 \cdot \operatorname{ones}(15,15)+0.2 \cdot I_{15}\right) \\
& W_{1}=-\left(\operatorname{hilb}(15)+0.2 \cdot \operatorname{ones}(15,15)-0.1 \cdot I_{15}\right) \\
& W_{2}=-\operatorname{hilb}(15)
\end{aligned}
$$

where hilb $(n)$ denotes the $n \times n$ Hilbert matrix, ones $(m, n)$ is the $m \times n$ matrix with all entries equal to one and $I_{n}$ is the identity matrix. Following Procedure 2 , we compute the $45 \times 45$ matrix $W$. The rank of this matrix is 36 but its reduced row echelon form $R$ obtained by the corresponding MATLAB function [14] has 42 leading 1's. Hence, $\operatorname{rank}(R)=42$ and consequently the realization will not be minimal. In fact, by applying Procedure $1, W=G U$ with

$$
\begin{aligned}
G & =[W(:, 1: 11) W(:, 13) W(:, 16: 45)] \in \mathbb{R}^{45 \times 42} \\
U & =\left[\begin{array}{ccccc|c|c}
I_{11} & V_{1} & O_{11 \times 1} & V_{2} & V_{3} & O_{11 \times 15} & O_{11 \times 15} \\
O_{1 \times 11} & 0 & 1 & \star & \star & O_{1 \times 15} & O_{1 \times 15} \\
O_{15 \times 11} & O_{15 \times 1} & O_{15 \times 1} & O_{15 \times 1} & O_{15 \times 1} & I_{15} & O_{15 \times 15} \\
O_{15 \times 11} & O_{15 \times 1} & O_{15 \times 1} & O_{15 \times 1} & O_{15 \times 1} & O_{15 \times 15} & I_{15}
\end{array}\right] \in \mathbb{R}^{42 \times 45}
\end{aligned}
$$


where $V_{i} \in \mathbb{R}^{11 \times 1}, i=1,2,3$, and the stars mean nonzero entries.

But $U$ has not the structure given by (2). We need to permute the columns twelve and thirteen by means of the permutation matrix $P=\operatorname{diag}\left(P_{1} P_{1} P_{1}\right)$ where $P_{1}=\left[\begin{array}{llllllll}e_{1} & e_{2} & \ldots & e_{11} & e_{13} & e_{12} & e_{14} & e_{15}\end{array}\right]$ and then, the rows twenty four and twenty five and the rows thirty-nine and forty premultiplying by $Z=$

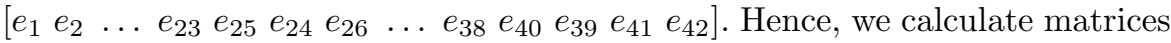
$\bar{G}=G Z^{T}$ and

$$
\bar{U}=Z U P=\left[\begin{array}{ccccccc}
I_{11} & O_{11 \times 1} & V_{1} & V_{2} & V_{3} & O_{11 \times 15} & O_{11 \times 15} \\
O_{1 \times 11} & 1 & 0 & \star & \star & O_{1 \times 15} & O_{1 \times 15} \\
O_{15 \times 11} & O_{15 \times 1} & O_{15 \times 1} & O_{15 \times 1} & O_{15 \times 1} & I_{15} & O_{15 \times 15} \\
O_{15 \times 11} & O_{15 \times 1} & O_{15 \times 1} & O_{15 \times 1} & O_{15 \times 1} & O_{15 \times 15} & I_{15}
\end{array}\right],
$$

such that $W P=\bar{G} \bar{U}$. Now, by Procedure 2, we compute the realization $(N, I, B, C)$ with

$$
\begin{aligned}
& B=\left[\begin{array}{c}
O_{11 \times 15} \\
O_{1 \times 15} \\
O_{15 \times 15} \\
I_{15}
\end{array}\right] \cdot P_{1}^{T} \\
& C=\bar{G}(1: 15,:) \\
& N=\left[\begin{array}{ccccccc}
O_{11 \times 12} & I_{11} & O_{11 \times 1} & V_{1} & V_{2} & V_{3} & O_{11 \times 15} \\
O_{1 \times 12} & O_{1 \times 11} & 1 & 0 & \star & \star & O_{1 \times 15} \\
O_{15 \times 12} & O_{15 \times 11} & O_{15 \times 1} & O_{15 \times 1} & O_{15 \times 1} & O_{15 \times 1} & I_{15} \\
O_{15 \times 12} & O_{15 \times 11} & O_{15 \times 1} & O_{15 \times 1} & O_{15 \times 1} & O_{15 \times 1} & O_{15 \times 15}
\end{array}\right] .
\end{aligned}
$$

This realization represents a system whose controllability matrix has rank 42 but its observability matrix has rank 36 . Therefore, this system is controllable but not observable. Thus, the realization is not minimal.

However, note that this fact, despite being important from a numerical point of view, it is irrelevant if we consider the implementation objectives. Indeed, it is intended to be applied to solve two classical problems in control theory: the model reduction problem in singular systems and the positive realization problem in linear control systems, being impossible for both of them to reach the minimum structure. Nevertheless, reducing the number of system variables or to obtaining a positive realization can lead to a significant improvement and reduction in the number of calculations.

Finally, taking into account the following nilpotent matrix of nilpotent index $t$ :

$$
J_{t, \alpha}=\left[\begin{array}{ccccc}
O & O & \ldots & O & O \\
I_{\alpha} & O & \ldots & O & O \\
O & I_{\alpha} & \ldots & O & O \\
\vdots & \vdots & & \vdots & \vdots \\
O & O & \ldots & I_{\alpha} & O
\end{array}\right] \in \mathbb{R}^{t \alpha \times t \alpha}
$$

from Procedure 2, the next result can be deduced. 
Proposition 2 Consider $P(s)=W_{0}+W_{1} s+\cdots+W_{t-1} s^{t-1} \in \mathbb{R}^{m \times p}(s)$.

(1) If $\operatorname{rank}\left(W_{t-1}\right)=p$ then a minimal realization $\left(N, I_{t p}, B, C\right)$ of $P(s)$ with a nilpotent matrix $N \in \mathbb{R}^{t p \times t p}, B \in \mathbb{R}^{t p \times p}$ and $C \in \mathbb{R}^{m \times t p}$ is given by

$$
N=J_{t, p}, \quad B=\left[\begin{array}{c}
I_{p} \\
O \\
\vdots \\
O
\end{array}\right] \quad \text { and } \quad C=\left[\begin{array}{llll}
-W_{0}-W_{1} & \cdots & -W_{t-2} & -W_{t-1}
\end{array}\right]
$$

(2) If $\operatorname{rank}\left(W_{t-1}\right)=m$ then a minimal realization $\left(N, I_{t m}, B, C\right)$ of $P(s)$ with a nilpotent matrix $N \in \mathbb{R}^{t m \times t m}, B \in \mathbb{R}^{t m \times p}$ and $C \in \mathbb{R}^{m \times t m}$ is given by

$$
N=J_{t, m}^{T}, \quad B=\left[\begin{array}{c}
-W_{0} \\
-W_{1} \\
\vdots \\
-W_{t-2} \\
-W_{t-1}
\end{array}\right] \quad \text { and } \quad C=\left[\begin{array}{lllll}
I_{m} & O & \cdots & O & O
\end{array}\right]
$$

Example 3 Consider the polynomial matrix

$$
\begin{aligned}
P(s) & =-\left[\begin{array}{ccc}
s+1 & s^{2}+2 s+2 & 2 s^{2}+6 s+3 \\
2 & 2 s^{2}+s+1 & 4 s^{2}+4 s \\
s+1 & s^{2} & 2 s^{2}+2 s+2
\end{array}\right]= \\
& =-\left[\begin{array}{lll}
1 & 2 & 3 \\
2 & 1 & 0 \\
1 & 0 & 2
\end{array}\right]-\left[\begin{array}{lll}
1 & 2 & 6 \\
0 & 1 & 4 \\
1 & 0 & 2
\end{array}\right] s-\left[\begin{array}{lll}
0 & 1 & 2 \\
0 & 2 & 4 \\
0 & 1 & 2
\end{array}\right] s^{2}= \\
& =W_{0}+W_{1} s+W_{2} s^{2} .
\end{aligned}
$$

To compute a minimal realization of $P(s)$ we define the matrix

$$
W=\left[\begin{array}{ccc}
W_{2} & W_{1} & W_{0} \\
O & W_{2} & W_{1} \\
O & O & W_{2}
\end{array}\right], \quad \text { with } \quad m=p=t=3
$$

Note that $W$ is the matrix $M$ of Example 1. Then, by using matrices

$$
\begin{aligned}
& P=\operatorname{diag}\left(\begin{array}{lll}
P_{1} & P_{1} & P_{1}
\end{array}\right), \quad \text { with } P_{1}=\left[\begin{array}{lll}
0 & 1 & 0 \\
1 & 0 & 0 \\
0 & 0 & 1
\end{array}\right] \text { and } \\
& Z=\left[\begin{array}{llllll}
e_{1} & e_{3} & e_{2} & e_{5} & e_{4} & e_{6}
\end{array}\right],
\end{aligned}
$$


we obtain the full rank factorization of $W P$,

$$
W P=\left[\begin{array}{llllll}
1 & 2 & 1 & 2 & 1 & 3 \\
2 & 1 & 0 & 1 & 2 & 0 \\
1 & 0 & 1 & 0 & 1 & 2 \\
0 & 1 & 0 & 2 & 1 & 6 \\
0 & 2 & 0 & 1 & 0 & 4 \\
0 & 1 & 0 & 0 & 1 & 2 \\
0 & 0 & 0 & 1 & 0 & 2 \\
0 & 0 & 0 & 2 & 0 & 4 \\
0 & 0 & 0 & 1 & 0 & 2
\end{array}\right]\left[\begin{array}{lllllll|lll}
1 & 0 & 2 & 0 & 0 & 1 & 0 & 0 & 0 \\
0 & 0 & 0 & 1 & 0 & 2 & 0 & 0 & 0 \\
0 & 0 & 0 & 0 & 1 & 1 & 0 & 0 & 0 \\
0 & 0 & 0 & 0 & 0 & 0 & 1 & 0 & 0 \\
0 & 0 & 0 & 0 & 0 & 0 & 0 & 1 & 0 \\
0 & 0 & 0 & 0 & 0 & 0 & 0 & 0 & 1
\end{array}\right]=\bar{G} \bar{U}
$$

Consequently, by Procedure 2

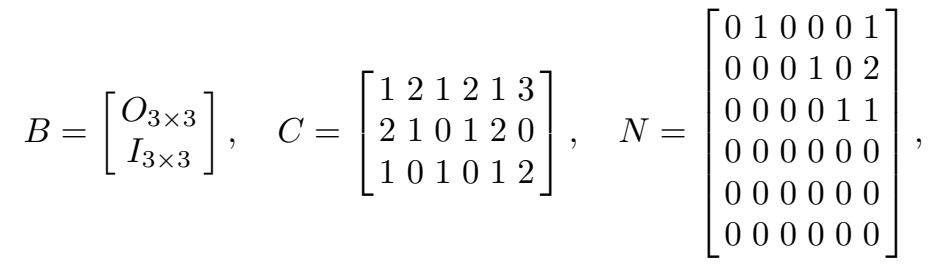

and $\left(N, I, B P_{1}^{T}, C\right)$ is a minimal realization of the polynomial matrix $P(s)$.

\section{Application to Singular Systems}

Singular systems have been one of the principal research topics in control theory for the last 50 years by their applications in many different areas such as the Leontief dynamic model, computer network, electrical, mechanical or communication systems and even, in biological systems. They are also called as descriptor systems, differential-algebraic systems or generalized state-space systems in the literature $[3,17]$.

A singular system can be represented by the next space-state model

$$
\left\{\begin{array}{l}
E \dot{x}(t)=A x(t)+B u(t) \\
y(t)=C x(t)
\end{array}\right.
$$

where $\dot{x}(t)=\frac{d x(t)}{d t}, t$ is the time, $x(t) \in \mathbb{R}^{n}$ is the vector of internal variables, $u(t) \in \mathbb{R}^{p}$ is the vector of control inputs and $y(t) \in \mathbb{R}^{m}$ is the vector of outputs and $E \in \mathbb{R}^{n \times n}$ is a singular matrix. If $E=I_{n}$ the identity matrix, we have a standard system.

But singular systems can also be defined by an input-output model given by a rational matrix $G(s)$. Both models are interchangeable. The matrices $E, A \in \mathbb{R}^{n \times n}, B \in \mathbb{R}^{n \times p}$ and $C \in \mathbb{R}^{m \times n}$ such that

$$
G(s)=C(s E-A)^{-1} B
$$

constitute a realization of $G(s)$, which is denote by $(E, A, B, C)$. The size of $A$ is called the dimension of the realization that is minimal if it has minimum dimension. 
Theorem 1 [7, pp 60] Any $m \times p$ rational matrix $G(s)$ may be represented as $G(s)=H(s)+P(s)$ where $H(s)$ is a strictly proper rational matrix and $P(s)$ is a polynomial matrix.

Theorem 1 implies that system (3) is equivalent to the canonical forwardbackward form given by

$$
\left\{\begin{array}{l}
\bar{E} \dot{\bar{x}}(t)=\bar{A} \bar{x}(t)+\bar{B} \bar{u}(t) \\
\bar{y}(t)=\bar{C} \bar{x}(t)
\end{array}\right.
$$

with $\bar{E}=\operatorname{diag}\left(I_{n_{1}}, N\right), \bar{A}=\operatorname{diag}\left(A_{1}, I_{n_{2}}\right), n_{1}+n_{2}=n$, where $n_{1}$ is the degree of polynomial $\operatorname{det}(s E-A), A_{1} \in \mathbb{R}^{n_{1} \times n_{1}}$ and $N \in \mathbb{R}^{n_{2} \times n_{2}}$ is a nilpotent matrix with index $t ; \bar{B}=B_{1} \oplus B_{2}$ with $B_{1} \in \mathbb{R}^{n_{1} \times p}$ and $B_{2} \in \mathbb{R}^{n_{2} \times p}$, and $\bar{C}=C_{1} \oplus C_{2}$ with $C_{1} \in \mathbb{R}^{m \times n_{1}}$ and $C_{2} \in \mathbb{R}^{m \times n_{2}}$. Therefore, its transfer matrix is the sum of the strictly proper rational matrix

$$
H(s)=C_{1}\left(s I_{n_{1}}-A_{1}\right)^{-1} B_{1}
$$

and the polynomial matrix

$$
P(s)=C_{2}\left(s N-I_{n_{2}}\right)^{-1} B_{2}
$$

So, Procedure 2 could be useful for singular systems. Two direct applications of this method are shown below.

\subsection{Model reduction}

As mentioned earlier, an application of singular systems is found in the study of electrical systems. Specifically, the dynamics of an electrical system can be described in terms of the differential equations (3), which are obtained by applying Kirchoff's laws. However, the matrices $E, A, B$ and $C$ usually have large sizes and are quite sparse, which means that some of the corresponding equations are redundant Unfortunately, for large-scale linear systems, the determination of a minimum set of equations and, therefore, a minimum number of variables, can be computationally non-trivial.

Procedure 2 can help us with the model reduction problem, as shown in the following example.

Example 4 Let the linear RLC circuit (a circuit with linear resistors, inductors and capacitors, see [7] for details) given by (3) where $E=\left(e_{i j}\right) \in \mathbb{R}^{11 \times 11}$ is 
the zero matrix except for $e_{22}=e_{99}=1$,

$$
A=\left[\begin{array}{rrrrrrrrrrr}
-1 & 0 & 0 & 0 & 0 & 0 & 0 & 0 & 0 & 0 & 0 \\
0 & 0 & 0 & 0 & 0 & 1 & 1 & 0 & 0 & 0 & 0 \\
0 & 0 & -1 & 0 & 0 & 0 & 1 & 1 & 0 & 0 & 0 \\
1 & 0 & 0 & 0 & 0 & 0 & 0 & 0 & 0 & 0 & -1 \\
0 & 1 & 0 & 0 & 0 & 0 & 0 & 0 & 0 & 0 & -3 \\
0 & 0 & 1 & 0 & 0 & 0 & 0 & 0 & 0 & 0 & -1 \\
0 & 0 & 0 & 1 & 0 & 0 & 0 & 0 & 0 & 0 & -1 \\
0 & 0 & 0 & 0 & 1 & 0 & 0 & 0 & 0 & 0 & -1 \\
0 & 0 & 0 & 1 & 0 & 0 & 0 & 0 & 0 & 0 & 0 \\
0 & 0 & 0 & 0 & 0 & 0 & 0 & 0 & 0 & -1 & 0 \\
0 & 0 & 0 & 0 & 0 & -1 & -3 & -1 & -1 & -1 & 0
\end{array}\right] \in \mathbb{R}^{11 \times 11}
$$

$B=\left(b_{i j}\right) \in \mathbb{R}^{11 \times 2}$ is the zero matrix except for $b_{11}=b_{10,2}=1$ and $C=I_{11}$ is the identity matrix.

It is easy to see that this system can be reduced, since $E, A$ and $B$ are sparse matrices. For model reduction, we compute its transfer matrix and try to obtain a minimal realization. According to Theorem $1, G(s)$ can be represented as follows:

$$
\begin{aligned}
G(s) & =\bar{C}[s \bar{E}-\bar{A}]^{-1} \bar{B}=G_{1}(s)+G_{2}(s)= \\
& =\frac{1}{s}\left[\begin{array}{lllllllllll}
0 & 0 & 0 & 0 & 0 & 1 & -1 & 1 & 1 & 0 & 0 \\
0 & 0 & 0 & 0 & 0 & 0 & 0 & 0 & 0 & 0 & 0
\end{array}\right]^{T}+ \\
& +\left[\begin{array}{rrrrrrrrrrrrr}
1 & 3 & 1 & 1 & 1 & 6 & s+1 & -3 & s-1 & 3 s+2 & 0 & 0 & 1 \\
0 & 0 & 0 & 0 & 0 & 1 & -1 & 1 & 0 & 1 & 0
\end{array}\right]^{T} .
\end{aligned}
$$

By [4], a minimal realization $\left(A_{1}, B_{1}, C_{1}\right)$ of $G_{1}(s)$ is

$$
A_{1}=[0] \quad B_{1}=\left[\begin{array}{ll}
1 & 0
\end{array}\right] \quad C_{1}=\left[\begin{array}{lllllllllll}
0 & 0 & 0 & 0 & 0 & 1 & -1 & 1 & 1 & 0 & 0
\end{array}\right]^{T},
$$

and by Procedure 2, a minimal realization $\left(N, I, B_{2}, C_{2}\right)$ of $G_{2}(s)$ is computed. Let

$$
\begin{aligned}
& G_{2}(s)=W_{1} s+W_{0}= \\
& =s\left[\begin{array}{rrrrrrrrrrr}
0 & 0 & 0 & 0 & 0 & 6 & -3 & 3 & 0 & 0 & 0 \\
0 & 0 & 0 & 0 & 0 & 0 & 0 & 0 & 0 & 0 & 0
\end{array}\right]^{T}+ \\
& +\left[\begin{array}{lllllllllll}
1 & 3 & 1 & 1 & 1 & 1 & -1 & 2 & 0 & 0 & 1 \\
0 & 0 & 0 & 0 & 0 & 1 & -1 & 1 & 0 & 1 & 0
\end{array}\right]^{T} .
\end{aligned}
$$

The matrix $W=\left[\begin{array}{cc}-W_{1} & -W_{0} \\ O & -W_{1}\end{array}\right]$ has the following full rank factorization

$$
W=G * U=[W(:, 1) W(:, 3: 4)]\left[\begin{array}{cc|cc}
1 & 0 & 0 & 0 \\
0 & 0 & 1 & 0 \\
0 & 0 & 0 & 1
\end{array}\right]
$$


where $U$ is a basic upper block matrix. Therefore, applying Procedure 2 we obtain

$$
\begin{gathered}
B_{2}=U(:, 3: 4)=\left[\begin{array}{ll}
0 & 0 \\
1 & 0 \\
0 & 1
\end{array}\right] \\
C_{2}=G(1: 11,:)=\left[\begin{array}{rrrrrrrrrr}
0 & 0 & 0 & 0 & 0-6 & -3 & 0 & 0 & 0 \\
-1 & -3-1-1-1-1 & 1-2 & 0 & 0 & -1 \\
0 & 0 & 0 & 0 & 0-1 & 1-1 & 0 & -1 & 0
\end{array}\right]^{T} \\
q_{t-1}=q_{1}=1, q_{t-2}=q_{0}=2, \text { then } N=\left[O_{3 \times 1} U(:, 1: 2)\right]=\left[\begin{array}{lll}
0 & 1 & 0 \\
0 & 0 & 0 \\
0 & 0 & 0
\end{array}\right] .
\end{gathered}
$$

From $\left(A_{1}, B_{1}, C_{1}\right)$ of $G_{1}(s)$ and $\left(N, I, B_{2}, C_{2}\right)$ of $G_{2}(s)$, a minimal realization $(E, A, B, C)$ of $G(s)$ is set up where

$$
\begin{aligned}
& E=\operatorname{diag}(1, N) \in \mathbb{R}^{4 \times 4} A=\operatorname{diag}\left(A_{1}, I\right) \in \mathbb{R}^{4 \times 4} \\
& B=\left[\begin{array}{l}
B_{1} \\
B_{2}
\end{array}\right] \in \mathbb{R}^{4 \times 2} \quad C=\left[C_{1} C_{2}\right] \in \mathbb{R}^{11 \times 4} .
\end{aligned}
$$

It must be noted that we have started with a space-state representation of order 11 but we have found a minimal realization of order 4 , significantly reducing the number of variables of this electrical circuit.

4.2 An interesting contribution to the positive realization problem

The importance of the positive realization problem in standard systems is well documented in diverse applicative fields. It appears, for instance, in the identification of compartmental systems, in the filtering of data generated by hidden Markov sequences or in the design of digital filters $[2,4,6,12,15]$. It is formulated as follows: Let $H(s) \in \mathbb{R}^{m \times p}(s)$ be a rational transfer matrix. It is said to admit a positive realization $(A, B, C)$ if we find a Metzler matrix $A \in \mathbb{R}^{n \times n}$, and nonnegative matrices $B \in \mathbb{R}^{n \times p}, C \in \mathbb{R}^{m \times n}$ such that $H(s)=$ $C(s I-A)^{-1} B$. Moreover, this realization is minimal if it has the minimum dimension.

In this section we show how Procedure 2 can be used to obtain positive realizations for rational transfer matrices with multiple real poles. In order to do so, the rational transfer matrix must be rewritten based on a polynomial matrix and then apply Proposition 2.

Proposition 3 Let $H(s)$ be the rational matrix

$$
H(s)=\sum_{i=0}^{t-1} \frac{M_{i}}{(s-a)^{i+1}}
$$


with $\operatorname{rank}\left(M_{i}\right)=p$ or $\operatorname{rank}\left(M_{i}\right)=m$. If $M_{i} \in R_{+}^{m \times p}$, for $i=0,1, \ldots, t-1$ then $H(s)$ admits a positive realization.

Proof Let us recall that we want to find a Metzler matrix $A$ and nonnegative matrices $B, C$, such that $H(s)=\sum_{i=0}^{t-1} \frac{M_{i}}{(s-a)^{i+1}}=C(s I-A)^{-1} B$.

However, any transfer matrix can be reformulated as follows:

$$
\begin{aligned}
H(s) & =C(s I-A)^{-1} B=C(\underbrace{(s-a)}_{h} I-\underbrace{(A-a I)}_{N})^{-1} B=C(h I-N)^{-1} B= \\
& =C\left(h\left(I-\frac{1}{h} N\right)\right)^{-1} B=\underbrace{\frac{1}{h}}_{q} C\left(I-\frac{1}{h} N\right)^{-1} B= \\
& =q C(I-q N)^{-1} B=-q C(q N-I)^{-1} B=-q P(q)
\end{aligned}
$$

with $N$ a nilpotent matrix of index $t$ and where $P(q)$ is a polynomial matrix. As $\operatorname{rank}\left(M_{i}\right)=p$ or $\operatorname{rank}\left(M_{i}\right)=m$, and $M_{i} \in R_{+}^{m \times p}$, for $i=0,1, \ldots, t-1$, by Proposition 2 we can obtain a realization $(N, I, B, C)$ of $P(q)$ with $N \geq O$, $B \geq O$ and $C \geq O$. Therefore, $A=N+a I$ is a Metzler matrix and $(A, B, C)$ a positive realization of $H(s)$ for all $a \in R$.

Example 5 Consider the transfer matrix

$$
\begin{aligned}
H(s) & =\frac{1}{(s-3)^{3}}\left[\begin{array}{cc}
s^{2}-4 s+4 s^{2}-s+1 \\
s-2 & s \\
s^{2}+3 s-5 s^{2}+s-5
\end{array}\right]= \\
& =\frac{1}{s-3}\left[\begin{array}{ll}
1 & 1 \\
0 & 0 \\
1 & 1
\end{array}\right]+\frac{1}{(s-3)^{2}}\left[\begin{array}{ll}
2 & 5 \\
1 & 1 \\
9 & 7
\end{array}\right]+\frac{1}{(s-3)^{3}}\left[\begin{array}{rr}
1 & 7 \\
1 & 3 \\
13 & 7
\end{array}\right] .
\end{aligned}
$$

This transfer matrix admits a positive realization by Proposition 3. Following the proof of this proposition, we are going to be able to construct this realization. Hence, considering $q=\frac{1}{s-3}$, we can write $H(s)$ by means of a polynomial matrix $P(q)$, that is:

$$
\begin{aligned}
H(s) & =-q\left(\left[\begin{array}{rr}
-1 & -1 \\
0 & 0 \\
-1 & -1
\end{array}\right]+q\left[\begin{array}{l}
-2-5 \\
-1-1 \\
-9-7
\end{array}\right]+q^{2}\left[\begin{array}{rr}
-1 & -7 \\
-1 & -3 \\
-13-7
\end{array}\right]\right)= \\
& =-q\left(W_{0}+q W_{1}+q^{2} W_{2}\right) .
\end{aligned}
$$

Now, by Proposition 2, the realization $(N, B, C)$ with

$$
B=\left[\begin{array}{l}
I_{2 \times 2} \\
O_{4 \times 2}
\end{array}\right], \quad C=\left[\begin{array}{lll}
W_{0} W_{1} W_{2}
\end{array}\right], \quad N=\left[\begin{array}{llllll}
0 & 0 & 0 & 0 & 0 & 0 \\
0 & 0 & 0 & 0 & 0 & 0 \\
1 & 0 & 0 & 0 & 0 & 0 \\
0 & 1 & 0 & 0 & 0 & 0 \\
0 & 0 & 1 & 0 & 0 & 0 \\
0 & 0 & 0 & 1 & 0 & 0
\end{array}\right]
$$


is obtained and finally, taking

$$
A=N+3 I=\left[\begin{array}{llllll}
3 & 0 & 0 & 0 & 0 & 0 \\
0 & 3 & 0 & 0 & 0 & 0 \\
1 & 0 & 3 & 0 & 0 & 0 \\
0 & 1 & 0 & 3 & 0 & 0 \\
0 & 0 & 1 & 0 & 3 & 0 \\
0 & 0 & 0 & 1 & 0 & 3
\end{array}\right],
$$

the positive realization $(A, B, C)$ of $H(s)$ is computed.

Remark 3 When a transfer matrix has different multiple real poles it is possible to obtain a positive realization if it can be decomposed in as many rational matrices that satisfied Proposition 3 as different poles.

\section{References}

1. Anderson, B. D. O. and Bongpanitlerd, S.: Network Analysis and Synthesis, A Modern Systems Theory Approach. Prentice-Hall, Inc., New Jersey, (1968).

2. Benvenuti, L. and Farina, L.: A Tutorial on the Positive Realization Problem. IEEE Transactions on Automatic Control, 49(5), 651-664, (2004). DOI: 10.1109/TAC.2004.826715

3. Bru, R., Coll, C. and Sánchez, E.: Structural properties of positive linear time-invariant difference-algebraic equations. Linear Algebra and its Applications, 349, 1-10, (2002). https://doi.org/10.1016/S0024-3795(02)00277-X

4. Cantó, R., Ricarte, B. and Urbano, A. M.: Positive Realizations of Transfer Matrices with real poles. IEEE Trans. Circuits Syst. II, Expr. Briefs, 54(6), 517-521, (2007). DOI: 10.1109/TCSII.2007.894408

5. Cantó, R., Ricarte, B. and Urbano, A. M.: On Positivity of Discrete-Time Singular Systems and the Realization Problem. Lecture Notes in Control and Information Sciences, 389, 251-258, (2009). https://doi.org/10.1007/978-3-642-02894-6_24

6. Climent, J., Napp, D. and Requena, V.: Block Toeplitz matrices for burst-correcting convolutional codes. RACSAM 114, 38 (2020). https://doi.org/10.1007/s13398-019-00744$\mathrm{y}$

7. Dai, L.: Singular Control Systems. Lecture Notes in Control and Information Sciences, Springer-Verlag, New York, (1989).

8. Golub, G. H. and Van Loan, C. F.: Matrix Computations. Johns Hopkins University Press, Fourth edition, Baltimore, (2013).

9. Henrion, D. and Šebek, M.: Polynomial and matrix fraction description. Control Systems, Robotics and automation, 7, 211-231, (2009). Available online in http://www.eolss.net/Sample-Chapters/C18/E6-43-13-05.pdf.

10. Ho, B. L. and Kalman, R. E.: Effective construction of linear state-variable models from mput/output functions. Regelungstechnik, 14(12),545-548, (1966).

11. Kaczorek, T.: Weakly positive continuous-time linear systems. Lecture Notes in Control and Information Sciences, 243, 3-16, (1999).

12. Kaczorek, T.: Positive 1D and 2D Systems, 431. Springer, London, (2002).

13. Kaczorek, T.: Externally and Internally Positive Singular Discrete-Time Linear Systems. International Journal of Appl. Math. Comput. Sci., 12(2), 197-202, (2002).

14. MATLAB, The Math Works, Inc., Natick, Massachusetts, United States. Official website: mathworks.com

15. McCrory, C., Parusinski, A.: The weight filtration for real algebraic varieties II: classical homology. RACSAM 108, 63-94 (2014). https://doi.org/10.1007/s13398-012-0098-y

16. Silverman, L.: Realization of linear dynamical systems. IEEE Transactions on Automatic Control, 16 (6), 554-567, (1971).

17. Virnik, E.: Stability analysis of positive descriptor systems. Linear Algebra and its Applications, 429(10), 2640-2659, (2008). 